\title{
Dynamic Melting and Decoupling of the Vortex Lattice in Layered Superconductors
}

\author{
Stefan Scheidl ${ }^{1,2}$ and Valerii M. Vinokur ${ }^{2}$ \\ ${ }^{1}$ Institut für Theoretische Physik, Universität zu Köln, Zülpicher Str. 77, D-50937 Köln, Germany \\ ${ }^{2}$ Materials Science Division, Argonne National Laboratory, Argonne, IL 60439
}

(April 30, 2018)

\begin{abstract}
The dynamic phase diagram of vortex lattices driven in disorder is calculated in two and three dimensions. A modified Lindemann criterion for the fluctuations of the distance of neighboring vortices is used, which unifies previous analytic approaches to the equilibrium and non-equilibrium phase transitions. The temperature shifts of the dynamic melting and decoupling transitions are found to scale inversely proportional to large driving currents. A comparison with two-dimensional simulations shows that this phenomenological approach can provide quantitative estimate for the location of these transitions.
\end{abstract}

PACS numbers: 74.25.Dw, 74.40.+k, 74.60.Ge

\section{INTRODUCTION}

The prediction of nonequilibrium phase transitions in driven vortex latticest has triggered an extensive theoretical study of periodic structures moving through a random-eprironment, using both analyticale $\mathrm{e}$ and numerical10 14 appraaches. The striking experiments on vortex transport 1521 that motivated and supported theoretical efforts, provided convincing evidence for a genuine nonequilibrium phase transition between different driven states of the vortex lattice. Investigations of the $I-V$ curves in $\mathrm{Nb}_{2}$ Se samples and MoGe films $15,18,19$ revealed regimes of plastic and elastic flow of the vortex lattice separated by a characteristic current. Neutron scattering experiments 17 on the driven vortex state in $\mathrm{Nb}_{2} \mathrm{Se}$ demonstrated a sharp increase in the density of structural defects of the vortex lattice in a certain current interval just above the depinning transition. At a larger characteristic current the defect density dropped significantly, suggesting a reordering of the vortex system.

To understand the above experiments it is crucial to determine how quenched disorder affects the structure of the driven vortex system. The central idea of Ref. 1 was the suggestion that disorder, being frozen in the laboratory frame, appears as a temporarily fluctuating force in the frame of the moving vortices and leads to an increased effective temperature of the vortex system. This effective temperature strongly depends on the drift velocity. Thus an equilibrium phase transition of the pure system (like melting) should have a counterpart in the system driven in a disordered environment and could be triggered by changing the drive at constant true temperature. For increasing drift velocities, disorder should be more and more washed out and the temperature of the dynamic transition should approach the transition temperature of the pure system from below.

One related question of principal interest is to what extent disorder-induced features of the equilibrium phase diagram could be eliminated by applying a driving current. The most prominent example for such a feature is the first-order melting transition of the vortex lattice, which is experimentally found to end at a critical point 22 For decreasing strength of disorder the location of the transition moves to higher temperatures and the end point wanders to larger fields.23 Therefore it seems plausible that with increasing sample purity this critical point continues to be shifted to larger fields and the theoretically expected phase diagram, which does not display a critical point, is recovered. Since it is practically impossible to obtain pinning-free samples, one might hope to observe the phase diagram of the ideal pure system by increasing a driving force in a given impure sample. The discussion of this scenario is one of the goals of the present paper.

Already in the pure case one has to face the problem of how to capture the melting transition theoretically. Here a phenomenological approach based on the Lindemann criterion has been used successfully 2428 This criterion states that the static lattice melts as the mean squared thermal displacement of a vortex line becomes equal to a certain fraction of the lattice spacing, $\left\langle\mathbf{u}^{2}(\mathbf{r}, t)\right\rangle \simeq c_{L}^{2} a_{0}^{2}$. The number $c_{L}$ is called the Lindemann number and is usually of order unity. $a_{0}$ is the vortex spacing in the direction perpendicular to the magnetic field. In this conventional form the Lindemann criterion would suggest that in two dimensions (where $\left\langle\mathbf{u}^{2}(\mathbf{r}, t)\right\rangle=\infty$ for all finite temperatures) crystals would always be unstable to thermal fluctuations. Indeed, the long-range translational order is lost, but a quasi-long-range translational order and the topological order persist at low temperatures and vanish only above a finite melting temperature. Since disorder in general reduces long-range order to quasi-longrange order between two an four dimensions, 2930 it is necessary to modify the phenomenological criterion for a detection of the more subtle loss of topological order. This is achieved by using a slightly modified criterion for the relative displacement of two neighbored vortices, 


$$
w(\mathbf{b})=\left\langle[\mathbf{u}(\mathbf{r}+\mathbf{b}, t)-\mathbf{u}(\mathbf{r}, t)]^{2}\right\rangle .
$$

Here $\mathbf{b}$ is a basis vector of the perfect lattice that separates the undisplaced vortices. If one naively thinks of the lattice as being built by vortices connected by springs, then $w$ is a measure for the typical stress of a bond ("spring") connecting neighboring vortices. Such bonds can be expected to "break" for

$$
w(\mathbf{b}) \approx c_{\mathrm{L}}^{2} a_{0}^{2} .
$$

In a layered superconductor, where the vortex lines are actually composed of point-like vortices, not only the melting transition but also a decoupling transition 27 can exist. The evaluation of bond widths $w$ for different orientations (in-plane of out-of-plane) provides additional insight into the anisotropy of vortex fluctuations and the location of these transitions.

The extension of the Lindemann criterion on disorderdominated system 31 enabled the description of disorderinduced static transitions between elastic and plastic glasses. In view of the loss of topological order the socalled "entanglement" transition from the Bragg glass to the vortex glass is equivalent to the melting of the pure system. Using the very same Lindemann criterion (2) with bond widths averaged over thermal and disorderinduced fluctuations, the location of this entanglement transition has been determined recently. 32 B The validity of this criterion has even been derived within a selfconsistent variational approach 32

For driven vortex systems disorder-induced displacements were calculated in Ref. 1 within a naive large velocity expansion approach for the two-dimensional vortex lattice, and the nonequilibrium melting line was found for the case of strong disorder. Based on scaling arguments Balents and Fisher extended the concept of nonequilibrium phase transitions over to charge density wave systems. 2 Giamarchi and Le Doussal 3 focused on the structure of the high velocity driven vortex phase and noticed that it retains some glassy features of itc static counterpart. The investigations that followed 4 国 60 revealed further fundamental features of the driven phase, in particular, the fact that in a coarse-grained description (i.e., on a large enough spatial scales) the main effect of disorder on the driven periodic structure can be described as a random force.

Most of these recent studies have been focused on the large-scale properties of the system in the elastic approximation. Based on the properties of the topologically constrained system it is in principle possible to include topological defects like dislocations and to study the topological stability of the lattice systematically. In practice this approach is difficult to realize, in particular when the transition appears as collective effect of the dislocations, as in the example of three-dimensional melting. Encouraged by the success of the Lindemann criterion in capturing the location of the phase transition in the pure case as well as in the static disordered case, we extend this approach in this paper to the location of nonequilibrium transitions of the vortex system.

The organization of this paper is as follows: in Sec. III we outline the perturbative approach for vortex lattices driven through disorder and specify the generalized form of Lindemann criterion. In Sec. III we evaluate the Lindemann criterion for two-dimensional systems and perform a quantitative comparison of the resulting phase diagram with numerical simulations. In Sec. IV we derive consequences for the dynamic phase diagram of threedimensional systems with a dynamic melting and decoupling transition in $\mathrm{V}$.

\section{PERTURBATIVE APPROACH}

We consider a vortex lattice in a steady driven state with velocity $\mathbf{v}$. Vortices are labeled by their ideal position $\mathbf{r}$ in the comoving frame, whereas their actual position in the laboratory frame is $\mathbf{R}(\mathbf{r}, t)=\mathbf{r}+\mathbf{v} t+\mathbf{u}(\mathbf{r}, t)$. The dynamics of the system is governed by the conventional equation for the vortex displacement field $\mathbf{u} \equiv$ $\mathbf{u}(\mathbf{r}, t)$ :

$$
\eta \dot{\mathbf{u}}=c \boldsymbol{\nabla}^{2} \mathbf{u}+\mathbf{f}^{\mathrm{pin}}(\mathbf{R})+\mathbf{F}-\eta \mathbf{v}+\boldsymbol{\zeta}
$$

where $c$ represents the elastic constants (specified below for the vortex lattice) and $\boldsymbol{\nabla}$ the lattice gradient. The thermal noise $\zeta$ couples the vortices to a heat bath of temperature $T$. The pinning force density $\mathbf{f}^{\operatorname{pin}}(\mathbf{r}, t)=$ $-\nabla V[\mathbf{R}(\mathbf{r}, t)]$ is related to the pinning potential $V$, which is supposed to be Gaussian distributed with a second moment

$$
\begin{aligned}
\overline{V(\mathbf{k}) V\left(\mathbf{k}^{\prime}\right)} & =\Delta(\mathbf{k}) \delta\left(\mathbf{k}+\mathbf{k}^{\prime}\right), \\
\Delta(\mathbf{k}) & =\Delta_{0} e^{-\frac{1}{2} k_{\perp}^{2} \xi^{2}} .
\end{aligned}
$$

The correlations decay on the scale of the coherence length $\xi$. We define $k_{\perp}^{2}=k_{x}^{2}+k_{y}^{2}$ as the vector component perpendicular to the magnetic field. Eq. (3) refers to point-like vortices in a single layer $(D=2)$ or in a layered superconductor $(D=3)$.

In the absence of disorder the response of the vortex lattice is different for longitudinal (L) and transverse (T) modes. Since the vortex lattice is almost incompressible, the longitudinal modes do not significantly contribute to many physical properties. However, as we see later on, in a vortex lattice driven through disorder these modes are important and have to be retained. The response function $G$ is determined by the elastic constants for compression $c_{11}$, shear $c_{66}$ and tilt $c_{44}$ :

$$
\begin{aligned}
G_{\alpha \beta}(\mathbf{q}, \omega) & =\sum_{p} G^{p}(\mathbf{q}, \omega) P_{\alpha \beta}^{p}(\mathbf{q}), \\
G^{p}(\mathbf{q}, \omega) & =\left[-i \eta \omega+c_{p} q_{\perp}^{2}+c_{44} q_{z}^{2}\right]^{-1}, \\
P_{\alpha \beta}^{T}(\mathbf{q}) & =\delta_{\alpha \beta}-\frac{q_{\alpha} q_{\beta}}{q_{\perp}^{2}}, \quad P_{\alpha \beta}^{L}(\mathbf{q})=\frac{q_{\alpha} q_{\beta}}{q_{\perp}^{2}},
\end{aligned}
$$


where $p=L, T$ stands for a polarizations with $c_{p}=$ $c_{11}, c_{66}$ respectively, and $q_{\perp}^{2}=q_{x}^{2}+q_{y}^{2}$. Wave vectors $\mathbf{q}$ are restricted to the first Brillouin zone (BZ) of the ideal lattice in contrast to $\mathbf{k}$.

To calculate the fluctuations $w$ of the distance of neighboring vortices we treat disorder on the lowest level of perturbation theory by approximating $\mathbf{f}(\mathbf{R})=\mathbf{f}(\mathbf{r}+\mathbf{v} t+$ $\mathbf{u}) \approx \mathbf{f}(\mathbf{r}+\mathbf{v} t)$, which is justified as long as $w(\mathbf{b}) \lesssim \xi^{2}$. This condition is satisfied in the whole range of interest $w(\mathbf{b}) \leq c_{L}^{2} a_{0}^{2}$ if $c_{L} a_{0} \lesssim \xi$. This restricts the validity of our approach to high magnetic fields not too much below the upper critical field.

In this approximation the pinning force can be considered as "external" force that does not depend on the response of the vortex lattice. The combination $\mathbf{f}(\mathbf{r}, t)=\mathbf{f}^{\mathrm{pin}}(\mathbf{r}+\mathbf{v} t)+\boldsymbol{\zeta}(\mathbf{r}, t)$ of this pinning force and the thermal noise has Gaussian correlations

$$
\begin{aligned}
\overline{\left\langle f_{\alpha}(\mathbf{q}, \omega) f_{\beta}\left(\mathbf{q}^{\prime}, \omega^{\prime}\right)\right\rangle} & =\Psi_{\alpha \beta}(\mathbf{q}, \omega) \delta\left(\mathbf{q}+\mathbf{q}^{\prime}\right) \delta\left(\omega+\omega^{\prime}\right), \quad(6 \mathrm{a}) \\
\Psi_{\alpha \beta}(\mathbf{q}, \omega) & =\vartheta \delta_{\alpha \beta}+\sum_{\mathbf{Q}} k_{\alpha} k_{\beta} \Delta(\mathbf{k}) \delta(\omega+\mathbf{v} \cdot \mathbf{k}), \\
\vartheta & =2 \eta T .
\end{aligned}
$$

The averaging includes the randomness of disorder and that of thermal noise. We denote $\mathbf{k}=\mathbf{Q}+\mathbf{q}$ with a reciprocal lattice vector (RLV) $\mathbf{Q}$ (being perpendicular to the magnetic field) and a vector $\mathbf{q}$ within the BZ.

The displacements in response to the total external force $\mathbf{f}$ have correlations

$$
\begin{aligned}
\overline{u_{\alpha}(\mathbf{q}, \omega) u_{\beta}\left(\mathbf{q}^{\prime}, \omega^{\prime}\right)} & =C_{\alpha \beta}(\mathbf{q}, \omega) \delta\left(\mathbf{q}+\mathbf{q}^{\prime}\right) \delta\left(\omega+\omega^{\prime}\right) \\
C_{\alpha \beta}(\mathbf{q}, \omega) & =G_{\alpha \gamma}(\mathbf{q}, \omega) \Psi_{\gamma \delta}(\mathbf{q}, \omega) G_{\beta \delta}(-\mathbf{q},-\omega) .
\end{aligned}
$$

From Eq. (7) we can immediately calculate the bond fluctuations

$$
w(\mathbf{b}) \approx \int_{\omega \mathbf{q}} \frac{1}{2}(\mathbf{q} \cdot \mathbf{b})^{2} C_{\alpha \alpha}(\mathbf{q}, \omega) .
$$

The thermal and pinning contributions to the force correlator (6) generate two corresponding contributions to the bond width, $w=w^{\text {th }}+w^{\text {pin }}$, which can be considered as approximately independent. The thermal contribution has been studied extensively in the past in order to determine the melting transition of the vortex lattice in the absence of impurities. Here we focus our attention on the contribution due to pinning, which is

$$
w^{\mathrm{pin}}(\mathbf{b}) \approx \sum_{p, \mathbf{Q}} \int_{\mathbf{q}} \frac{1}{2}(\mathbf{q} \cdot \mathbf{b})^{2} \Gamma^{p}(\mathbf{k}) \Delta(\mathbf{k})
$$

where $\int_{\mathbf{q}}=\int \frac{d^{D} q}{(2 \pi)^{D}}$ and we abbreviate

$$
\begin{aligned}
\Gamma^{T}(\mathbf{k}) & \equiv \frac{\left(\mathbf{k}_{\perp} \wedge \mathbf{q}_{\perp}\right)^{2}}{q_{\perp}^{2}} \frac{1}{\eta^{2}(\mathbf{v} \cdot \mathbf{k})^{2}+\left(c_{66} q_{\perp}^{2}+c_{44} q_{z}^{2}\right)^{2}}, \\
\Gamma^{L}(\mathbf{k}) & \equiv \frac{\left(\mathbf{k}_{\perp} \cdot \mathbf{q}_{\perp}\right)^{2}}{q_{\perp}^{2}} \frac{1}{\eta^{2}(\mathbf{v} \cdot \mathbf{k})^{2}+\left(c_{11} q_{\perp}^{2}+c_{44} q_{z}^{2}\right)^{2}}
\end{aligned}
$$

Before we proceed to a detailed evaluation of Eq. (9) in the following sections, we cite the result of lowest order perturbation theory for the macroscopic friction force $\mathbf{F}^{\text {fr }}$ arising from the collective summation of the microscopic pinning forces. In order to achieve a drift velocity $\mathbf{v}$ one has to apply a driving force $\mathbf{F}(\mathbf{v})=\eta \mathbf{v}+\mathbf{F}^{\mathrm{fr}}(\mathbf{v})$ with

$$
\begin{aligned}
F_{\alpha}^{\mathrm{fr}} & =\int_{\mathbf{k}} i k_{\alpha} k_{\beta} k_{\gamma} \Delta(\mathbf{k}) G_{\beta \gamma}(\mathbf{k},-\mathbf{v} \cdot \mathbf{k}) \\
& =\sum_{p, \mathbf{Q}} \int_{\mathbf{q}} k_{\alpha} \eta(\mathbf{v} \cdot \mathbf{k}) \Gamma^{p}(\mathbf{k}) \Delta(\mathbf{k}),
\end{aligned}
$$

which coincides for $D=2$ with the early result of Schmid and Hauger. 39

The disordered vortex lattice is characterized by two elementary length scales: the disorder correlation length $\xi$ and the "vortex spacing" $a_{0} \equiv \sqrt{\Phi_{0} / B}$ ( $B$ is the magnetic induction and $\Phi_{0}$ the flux quantum). In the triangular lattice the actual distance between neighboring vortices in a direction perpendicular to the magnetic field is $a=\sqrt{2 / \sqrt{3}} a_{0}$. We assume $c_{L} a_{0} \lesssim \xi \lesssim a_{0}$ which is realistic for high temperature superconductors at large fields. For the evaluation of the main formulae (9) and (11) we will retain the reciprocal lattice structure in $\mathbf{Q}$, but we approximate the BZ as spherical cylinder with bounds $\mathbf{q}_{\perp}^{2} \leq q_{\perp}^{* 2} \equiv 4 \pi / a_{0}^{2}$ and $\left|q_{z}\right| \leq q_{z}^{*} \equiv \pi / d$ with the layer spacing $d$. This approximation preserves the area of the BZ. The nonlocality of $c_{66}$ will be neglected. We choose the $x$-axis as the direction of the velocity, $\mathbf{v}=v \hat{\mathbf{x}}$, and suppose the vortex lattice to move along one main direction of the hexagonal lattice, which are the directions of minimum energy dissipation. 39

\section{EVALUATION IN $D=2$}

The general expressions for bond widths $w$ and friction force $\mathbf{F}^{\mathrm{fr}}$ are immediately specialized to two dimensions by setting $q_{z}=0$. They are evaluated here in order to compare the location of the dynamic melting transition according to the Lindemann criterion with numerical simulations.

\section{A. Thermal bond width}

To start with we consider the contribution of thermal fluctuations to the bond width. By a comparison with the Kosterlitz-Thouless melting theory 40 we are able to fix the Lindemann number $c_{L}$.

Thermal fluctuations lead to a displacement correlation

$$
C_{\alpha \alpha}^{\mathrm{th}}(\mathbf{q}, \omega)=\frac{\vartheta}{\eta^{2} \omega^{2}+c_{66}^{2} q^{4}},
$$


which implies a bond width

$$
w^{\mathrm{th}}(\mathbf{b}) \approx \int_{\omega \mathbf{q}} \frac{1}{2}(\mathbf{q} \cdot \mathbf{b})^{2} C_{\alpha \alpha}^{\mathrm{th}}(\mathbf{q}, \omega) \approx b^{2} \frac{T}{4 a_{0}^{2} c_{66}} .
$$

Here the contribution of longitudinal displacements can be safely neglected since typically $c_{11} \gg c_{66}$. In $D=2$ there is only one bond length $b=a$. The actua melting temperature within Kosterlitz-Thouless theory 40 (neglecting renormalization effects) is given by

$$
T_{\mathrm{KT}} \approx \frac{a_{0}^{2} c_{66}}{4 \pi}
$$

Inserting this temperature into Eq. (13) one can estimate the Lindemann parameter

$$
c_{\mathrm{L}}^{2} \approx \frac{1}{6 \sqrt{3} \pi} \approx 0.03 .
$$

This value is in good agreement with typical values that empirically describe the melting transition in high temperature superconductors. We stick to this value in all quantitative considerations below.

As soon as we have determined the pinning bond widths we can estimate the location of the transition where bonds $\mathbf{b}$ become unstable according to the Lindemann criterion $w^{\text {th }}+w^{\text {pin }}=c_{L}^{2} a_{0}^{2}$. The transition temperature will be determined by

$$
T_{\mathrm{c}}=T_{\mathrm{KT}}-\frac{4 a^{2} c_{66}}{a_{0}^{2}} w^{\mathrm{pin}}
$$

as a function of the bond orientation and of velocity.

\section{B. Pinning bond width}

The evaluation of the disorder contributions (9) to the bond width is more subtle than the calculation of the friction force. In particular, since the velocity selects a particular direction, which is chosen parallel to the $x$-axis (called $x$-bonds), we have to distinguish bonds pointing parallel to the velocity and bonds that enclose an angle of 60 degrees with the $x$-axis (called $y$-bonds). Both types of bonds have a length $|\mathbf{b}|=a$. Due to the invariance of Eq. (9) under a reflection of components of $\mathbf{k}$, one can parameterize the bond width by two coefficients,

$$
w^{\text {pin }}(\mathbf{b})=w_{x}^{\text {pin }} b_{x}^{2} / b^{2}+w_{y}^{\text {pin }} b_{y}^{2} / b^{2} .
$$

These coefficients $w_{x}^{\text {pin }}$ and $w_{y}^{\text {pin }}$ can be calculated analytically for small and large velocities:

$$
\begin{aligned}
& w_{x}^{\text {pin }} \approx\left\{\begin{array}{l}
\frac{a^{2} \Delta_{0}}{\sqrt{8 \pi} a_{0} \xi^{3} \eta^{2} v^{2}}, \\
\frac{a^{2} a_{0}^{2} \Delta_{0}}{32 \pi^{2} \xi^{4} c_{66}^{2}}\left(1+\frac{3 \sqrt{2 \pi} \xi}{2 a_{0}}\right) \ln \left(\frac{4 \pi \xi c_{66}}{a_{0}^{2} \eta v}\right),
\end{array}\right. \\
& w_{y}^{\text {pin }} \approx\left\{\begin{array}{l}
\frac{a^{2} \Delta_{0}}{4 \sqrt{2 \pi} \xi^{3} \eta v c_{11}}, \\
\frac{a^{2} a_{0}^{2} \Delta_{0}}{32 \pi^{2} \xi^{4} c_{66}^{2}}\left(1+\frac{\sqrt{2 \pi} \xi}{2 a_{0}}\right) \ln \left(\frac{4 \pi \xi c_{66}}{a_{0}^{2} \eta v}\right) .
\end{array}\right.
\end{aligned}
$$

Upper and lower expressions hold for $v \gg c_{11} / a_{0} \eta$ and $v \ll \xi c_{66} / a_{0}^{2} \eta$ respectively.

In the limit of large velocities the contributions to leading order in $v$ and in small $\xi / a_{0}$ come only from RLV with $Q_{x}=0$, since then the denominator of the response function becomes small, $\eta^{2} v^{2} k_{x}^{2}+c_{p}^{2} q^{4}=\eta^{2} v^{2} q_{x}^{2}+c_{p}^{2} q^{4}$, and gives largest weight to small $q_{x}$. In the case of $w_{x}^{\text {pin }}$ the elastic interaction is negligible above a characteristic velocity $v \sim c_{p} / a_{0} \eta$. Then longitudinal and transverse modes are equally important and give contributions $\sim v^{-2}$. For $w_{x}^{\text {pin }}$ vortices therefore respond as if they were independent particles. In the case of $w_{y}^{\text {pin }}$ there is a qualitative difference between longitudinal and transverse modes. Transverse modes again give only contributions $\sim v^{-2}$, whereas now longitudinal modes give dominating contributions of order $\sim v^{-1}$. Thus at large velocities $y$-bonds are subject to much stronger fluctuations than $x$-bonds.

For small velocities the elastic interaction dominates the response of vortices and for $c_{11} \gg c_{66}$ longitudinal modes are in general negligible compared to the transverse modes. The small velocity regime is reached when in the denominator $\eta^{2} v^{2} k_{x}^{2}+c_{p}^{2} q^{4}$ of the response functions the first term is typically small compared to the second one, i.e. below a velocity $v \sim \xi c_{p} / a_{0}^{2} \eta$. To the leading order in small $v$ and $\xi / a_{0}$, RLV of all directions contribute equally the the bond widths, which diverge $\sim \ln (1 / v)$. The anisotropy does not vanish at small velocities, the prefactor of the logarithmic divergence depends (in subleading order in $\xi / a_{0}$, arising from contributions of RLV with $Q_{x}=0$ ) on the bond orientation. In contrast to the high velocity regime, $x$-bonds have stronger fluctuations than $y$-bonds at small velocities.

These results will be discussed in more detail later in section IIIE.

\section{Friction force}

In most experiments and simulations the driving force rather than the velocity is the parameter controlling the drift. Therefore we wish to express the bond widths as a function of the force and to evaluate the transport characteristics $\mathbf{v}(\mathbf{F})$ from the friction force (11). Since we assume that vortices drift along a basic lattice direction, the friction force is also parallel to velocity, $\mathbf{F}^{\text {fr }}\|\mathbf{v}\| \mathbf{x}$. Eq. (11) can be evaluated analytically in the limits of large and small velocities. In both cases the main contributions to the sum over RLV's come from $\mathbf{Q}$ with all possible orientations. Therefore the characteristic velocity separating the large and small velocity regimes for transverse and longitudinal modes are given by $v \sim \xi c_{66} / a_{0}^{2} \eta$ and $v \sim \xi c_{11} / a_{0}^{2} \eta$ respectively. We find 


$$
\begin{aligned}
F^{\mathrm{fr}} & \approx \sum_{p, \mathbf{Q}} \Delta(\mathbf{Q}) \frac{Q^{2} Q_{x}}{8 \pi c_{p}} \text { atn } \frac{4 \pi c_{p}}{Q_{x} a_{0}^{2} \eta v} \\
& \approx \begin{cases}\frac{\Delta_{0}}{\pi \xi^{4} \eta v} & \text { for } v \gg \xi c_{11} / a_{0}^{2} \eta, \\
\frac{3 a_{0}^{2} \Delta_{0}}{2(2 \pi)^{5 / 2} \xi^{5} c_{66}} & \text { for } v \ll \xi c_{66} / a_{0}^{2} \eta .\end{cases}
\end{aligned}
$$

Only for large velocities $v \gg \xi c_{11} / a_{0}^{2} \eta$ longitudinal and transverse modes contribute equally to the friction force. At velocities $v \ll \xi c_{11} / a_{0}^{2} \eta$ the transverse modes dominate since $c_{11} \gg c_{66}$. Note that the friction force enters the large velocity regime already for $v \gg \xi c_{11} / a_{0}^{2} \eta$, whereas the bond widths reach their corresponding regime only for $v \gg c_{11} / a_{0} \eta$.

\section{Numerical evaluation}

In order to illustrate the analytic results and to demonstrate the capability of the Lindemann approach to provide even a quantitative estimate for the location of dynamic phase transitions, we perform a numerical evaluation of the bond width and the friction force from Eqs. (9) and (11). The results are then compared with the simulation data in Ref. 1 .

For this purpose we specify the parameters as follows. In Ref. 1 the vortex spacing $a$ was used as length scale and $2 d \epsilon_{0} \equiv 2 d\left(\Phi_{0} / 4 \pi \lambda\right)^{2}$ as energy scale $(\lambda$ is the penetration depth, $d$ is the layer thickness). The time scale is set by $\eta=1$. From the melting temperature $T_{\mathrm{KT}} \approx 0.007$ of the pure system we find $c_{66} \approx 0.088$ according to Eq. (14). The vortex interaction chosen in Ref. 1 decays on the penetration length estimated by $\lambda \approx a$. The compression constant is obtained from $c_{11} \approx\left(16 \pi \lambda^{2} / a^{2}\right) c_{66} \approx 50 c_{66}$. 11 We furthermore identify $\xi \equiv r_{p}=0.2$ and refer to the data sets with a pinning strength $A=0.006$, a number of pinning centers $N_{p}=10^{4}$, and a number of vortices $N_{v}=400$. Then the disorder strength is $\Delta_{0}=\gamma_{U} / a_{0}^{2}=\left(N_{p} / N_{v} a_{0}^{2}\right)\left[\pi A\left(\xi / a_{0}\right)^{2}\right]^{2} \approx 1.42 \cdot 10^{-5}$.

Fig. 1 shows the pinning bond widths for $x$-bonds (full line) and $y$-bonds (long dashed line). The short dashed lines are a guide to the eyes representing an asymptotic decay $\sim v^{-2}$ for $x$-bonds and $\sim v^{-1}$ for $y$-bonds. This asymptotic regime is reached only for $v \gtrsim 100$. At small velocities $v \lesssim 0.01$ both bond widths diverge logarithmically. For large velocities, $y$-bonds have stronger fluctuations than $x$-bonds, as opposed to small velocities.

The friction force obtained from Eq. (11) is shown in Fig. 2 by the full line. The dashed line displays a $v^{-1}$ dependence, which is realized by $F^{\mathrm{fr}}$ for $v \gtrsim 100$. At small velocities, $v \lesssim 0.01, F^{\text {fr }}$ saturates at a finite value in accordance with Eq. (19b). The resulting transport characteristics $v(F)$ is shown in Fig. 3. The dashed line is the characteristics in the absence of pinning, which is shifted to the full line by the presence of disorder. This shift is practically constant in the small velocity regime. The dots represent the simulation data of Ref. 1 for the lowest temperature $(T=0.001)$ considered there. The agreement with the perturbative result is surprising for $F>F_{c} \approx 0.04$, where vortices move coherently $\left(F_{c}\right.$ corresponds to $f_{t}$ in Ref. (1). The regime of forces $F<F_{c}$ is beyond the validity range of our elastic approach.

Fig. A displays the resulting phase diagram, comparing the result of the Lindemann criterion (full line) to the simulation (dots). Above this transition line all bonds are stable, below the transition line the Lindemann criterion breaks the breaking bonds. In the displayed range of small velocities $x$-bonds are the least stable. Agreement between perturbation theory and simulations is given up to a factor of the order of unity, which is still quite favorable in view of the conceptual simplicity of the Lindemann approach and its sensitivity to changes e.g. of the Lindemann number. It is worthwhile to state that no fit parameters have been used in our numerical calculation.

\section{E. Discussion}

At this point we rest to discuss some specific properties of our results (18a and (18b) for the bond widths.

The first property is the strong anisotropy at large velocity, where the width of $x$-bonds scales like $\sim 1 / v^{2}$ independent of the elastic constants, whereas the $y$-bond width scales like $\sim 1 / v c_{11}$. This is true only for largest velocities $v \gg c_{11} / a_{0} \eta$, whereas for $v \ll c_{11} / a_{0} \eta$ the isotropy is essentially restored, i.e. $y$-bonds have qualitatively the same velocity dependence as $x$-bonds but the prefactors are still different. At large $v$ the physical origin of the anisotropy can be understood in a simple picture where vortices are considered as independent particles. Vortices neighbored in $x$-direction follow the same paths and are exposed to the same pinning forces. They experience the same force, but with a delay time $\Delta t=a / v$. Even in the absence of vortex interactions such purely time shifted forces give rise to bond fluctuations of a finite width only. Therefore the elastic interaction is irrelevant and these bond fluctuations are independent of elastic constants and decay proportional to $(\Delta t)^{2} \propto v^{-2}$. Vortices neighbored in directions not parallel to velocity always move on different trajectories and are exposed to essentially uncorrelated pinning forces. In the absence of interactions their typical relative distance would increase without limits in the direction perpendicular to $\mathbf{v}$ as in a diffusion process. In the lattice such a diffusive motion is prevented by the vortex interactions. Since their relative distance fluctuates mainly in $y$-direction, which is almost parallel to their distance, it is the compression modulus rather than the shear modulus that confines the bond fluctuations.

The anisotropy at large $v$ can be related to the anisotropy of the Larkin domain in the driven lattice 3.9 This domain is much longer in the direction parallel to the velocity than in the other directions, which means that relative displacements grow faster in direction perpendicular to $\mathbf{v}$ than parallel to $\mathbf{v}$. Our conclusion that 
$y$-bonds are less stable than $x$-bonds at large velocities is in agreement with a smectic structure of the drifting system, aligned parallel to $\mathbf{v}$.

As second distinct feature of the bond fluctuations, the divergence of the bond widths at small velocities deserves some explanation. This divergence, which is in general found for $D \leq 2$, implies that at zero velocity and for arbitrarily weak disorder all bonds are broken and the vortex lattice is destroyed, i.e. that the structure factor resembles that of a liquid. This result of the Lindemann criterion coincides surprisingly with that of more elaborate methods, e.g. renormalization group methods, 4243 which evaluate the large-scale correlations of the displacement field. The divergence of the bond width in our approach arises from the integration over small momenta q, i.e. from large scale fluctuations. From a principal point of view our perturbative treatment of disorder is not valid at largest scales, where higher order effects can no longer be neglected. Therefore the correctly found divergence of the bond width has to be considered as lucky circumstance.

Although the phenomenological Lindemann criterion is able to capture the position of a given transition, it can neither proof the existence of a transition nor can it tell us something about the nature of a transition. Although in $2 \mathrm{D}$ a phase transition can be observed in the density of lattice defects and, as a consequence in specific features of the transport characteristics 15.18 scaling argumenta 2 and numerical calculations of the structure factor 10.13 indicate that on large length scales free defects should exist, i.e. that the topological order of the lattice is lost. However, at present a rigorous characterization of the large-scale properties and examination of the effects of defects is still missing. Even if a true phase transition (like "solid-to-fluid") related to a qualitative change of the large correlations might be absent in 2D case, we expect a transition (like "liquid-to-gas") or at least a pronounced crossover at the location of the dynamic phase "transition" located by the Lindemann criterion, which in its generalized form used here probes small scale correlations. While these displacement fluctuations on small scales are related to the rate of generation of (initially bound) dislocation pairs, large scale fluctuations of the displacement field are relevant to decide whether dislocations remain bound in pairs or dissociate into free dislocations that destroy the topological order.

It is instructive to draw a comparison between the bond widths, which we examine here, and the shaking temperature $T_{\mathrm{sh}}$ introduced in Ref. 1. $T_{\mathrm{sh}}$ was defined from the correlator of the pinning force experienced by a single particle. It is therefore independent of elastic constants. If we compare the thermal and disorder contributions to the bond width, the latter could be expressed in terms of an effective "bond shaking temperature"

$$
T^{\mathrm{bsh}}(\mathbf{b}) \equiv \frac{4 a_{0}^{2} c_{66}}{b^{2}} w(\mathbf{b}) .
$$

This bond shaking temperature differs from $T_{\mathrm{sh}}$ in several respects. First of all it depends on the orientation of the bonds under consideration. At large velocities $T_{\mathrm{sh}}$ and $T^{\mathrm{bsh}}$ for $y$-bonds have the dependence $\sim v^{-1}$ in common. However they differ in the prefactor, which is independent of the elastic constants in the former case (since $T_{\mathrm{sh}}$ characterizes a single particle), but contains a prefactor $\sim c_{66} / c_{11}$ in the latter case (since $T^{\mathrm{bsh}}$ characterizes a relative displacement response). $T^{\mathrm{bsh}}$ is more similar to the coherent shaking temperature $T_{\mathrm{sh}}^{\mathrm{coh}}$ of Ref. 1, which was found to decay $\sim v^{-2}$ in agreement with $T_{\text {bsh }}$ for $x$-bonds (and even $y$-bonds in an incompressible lattice).

Speaking about effective temperatures, the attribute "shaking" should not be taken too literally, since even for $v=0$ the bond width $w^{\text {pin }}$ is finite and static, i.e. it has not temporal fluctuations. However, a snapshot of the displacements on small scales looks like that of a system without disorder but with a temperature increased by $T^{\text {bsh }}$. Since an effective temperature cannot be defined uniquely in a nonequilibrium situation anyway, we keep in the following the notion of bond fluctuations. Since these fluctuations are related to the fluctuations of the Peach-Koehler force acting on dislocations, we believe that the bond fluctuations are an appropriate measure for the relevance of dislocation (at least on not too large scales, see discussion above). The order-of-magnitude agreement on the location of the transition between the Lindemann criterion and simulation supports this picture.

\section{EVALUATION IN 3D}

In a layered three-dimensional superconductor the physics is even more rich than in two dimensions: besides the melting transition, where the structural order of the vortex lattice gets lost and its elastic moduli get renormalized to zero, an additional decoupling transition, where the conductivity perpendicular to the layers becomes ohmic and the effective Josephson coupling between the layers gets lost, can occur. We evaluate the bond fluctuations for layered superconductors in the driven disordered case and discuss the implications for the nonequilibrium counterpart of the equilibrium melting and decoupling transitions.

In bulk superconductors, in particular for anisotropic high-temperature superconductors, the elastic properties of the vortex lattice are somewhat intricate due to the dispersion of the elastic constants (mainly of $c_{44}$ and $c_{11}$ ). For completeness we cite the values 25.27

$$
\begin{aligned}
& c_{11}=\frac{\epsilon_{0}}{a_{0}^{2}} \frac{\lambda^{2} q_{\perp}^{* 2}}{1+\gamma^{2} \lambda^{2} q_{\perp}^{2}+\lambda^{2} q_{z}^{2}} \frac{1+\gamma^{2} \lambda^{2} q^{2}}{1+\lambda^{2} q^{2}}, \\
& c_{66}=\frac{\epsilon_{0}}{4 a_{0}^{2}}, \\
& c_{44}=c_{44}^{0}+c_{44}^{c},
\end{aligned}
$$




$$
\begin{aligned}
c_{44}^{0}= & \frac{\epsilon_{0}}{a_{0}^{2}} \frac{\lambda^{2} q_{\perp}^{*}}{1+\gamma^{2} \lambda^{2} q_{\perp}^{2}+\lambda^{2} q_{z}^{2}}, \\
c_{44}^{c}= & \frac{\epsilon_{0}}{2 a_{0}^{2}}\left[\frac{1}{\gamma^{2}} \ln \left(\frac{\gamma^{2} \xi^{-2}}{\lambda^{-2}+\gamma^{2} q_{\perp}^{*}+q_{z}^{2}}\right)\right. \\
& \left.+\frac{1}{\lambda^{2} q_{z}^{2}} \ln \left(1+\frac{\lambda^{2} q_{z}^{2}}{1+\lambda^{2} q_{\perp}^{*}}\right)\right] .
\end{aligned}
$$

We define $q_{\perp}^{*} \equiv \sqrt{4 \pi} / a_{0}, q_{z}^{*} \equiv \pi / d$, and $\epsilon_{0}=\left(\Phi_{0} / 4 \pi \lambda\right)^{2}$. The tilt modulus is composed of a nonlocal contribution to the tilt energy and a "single-vortex" contribution.27 We are interested in the range of not too small fields, where $\lambda \gtrsim a_{0}$ and an exponential decay of the elastic constants with the vortex density can be neglected.

\section{A. Thermal bond widths}

It is straightforward to evaluate the bond fluctuations due to thermal fluctuations and in the absence of disorder. Although purely thermal fluctuations have already been examined within the usual Lindemann approach 24 26,28, we calculate the bond fluctuations in order to gain additional insight into the anisotropic nature of the fluctuations. Neglecting again the contributions of compression modes, we find

$$
\begin{aligned}
w_{x}^{\text {th }} & \equiv w^{\text {th }}(a \hat{\mathbf{x}}) \approx \frac{a^{2} T}{4 d a_{0}^{2} c_{66}} \frac{\beta}{\sqrt{2}} \operatorname{atn}\left(\frac{\sqrt{2}}{\beta}\right) \\
& \approx \begin{cases}\frac{a^{2} T}{4 a_{0}^{2} d c_{66}} & \text { for } \beta \gg 1 \\
\frac{\pi a^{2} T}{8 \sqrt{2} a_{0}^{2} d c_{66}} \beta & \text { for } \beta \ll 1,\end{cases} \\
w_{z}^{\text {th }} & \equiv w^{\text {th }}(d \hat{\mathbf{z}}) \approx \frac{\pi T}{24 d c_{66}}\left[2 \beta^{2}-2 \beta^{3} \operatorname{atn} \frac{1}{\beta}+\ln \left(1+\beta^{2}\right)\right] \\
& \approx\left\{\begin{array}{lll}
\frac{\pi T}{12 d c_{66}} \ln \beta & \text { for } \beta \gg 1 \\
\frac{\pi T}{8 d c_{66}} \beta^{2} & \text { for } \beta \ll 1,
\end{array}\right.
\end{aligned}
$$

where

$$
\beta^{2} \equiv \frac{c_{66} q_{\perp}^{* 2}}{c_{44} q_{z}^{* 2}}=\frac{B}{B_{c r}}
$$

is the magnetic field in units of the crossover field 27, 44

$$
B_{c r}=\frac{\pi \Phi_{0} c_{44}}{4 d^{2} c_{66}} .
$$

For the transparency of our arguments and for qualitative purposes we have suppressed the explicit nonlocality of the elastic constants. The nonlocality can be restored by using effective values of the dispersive elastic constants (21) evaluated at the length scales that give the largest contributions to the integrals. In $D=3$ bond fluctuations are always dominated by small-scale fluctuations, since even in the presence of disorder and for small velocities one never encounters a divergence arising from large scales (unlike in $D \leq 2$ ). Therefore we use in our following estimates elastic constants evaluated for $q_{\perp}=q_{\perp}^{*}$ and $\left|q_{z}\right|=q_{z}^{*}$. In general this approximation underestimate the stiffness of the lattice but it is qualitatively valid as long as the dispersion (anisotropy of the elastic constants) is not too pronounced.

Let us now locate the lines where $w_{\alpha}^{\text {th }}=c_{l}^{2} a_{0}^{2}$ for all bond types $\alpha=x, y, z$. These lines can be described by $T_{\alpha} \equiv T_{\alpha}(B)$ in the $(T, B)$ plane. To be specific, we consider in the following moderately anisotropic systems with $\gamma d \ll \lambda$ (like for YBCO, and even BSCCO lies just at the border) at not too small fields $\left(a_{0} \leq \lambda\right)$. For such systems one finds at $q_{\perp}=q_{\perp}^{*}$ and $q_{z}=q_{z}^{*}$.27

$$
\begin{aligned}
c_{44} & \approx \frac{\Phi_{0}^{2}}{2\left(4 \pi \gamma a_{0} \lambda\right)^{2}} \ln \frac{\gamma^{2} / \xi^{2}}{4 \pi \gamma^{2} / a_{0}^{2}+\pi^{2} / d^{2}}, \\
B_{c r} & \approx \frac{\pi \Phi_{0}}{\gamma^{2} d^{2}} \ln (\gamma d / \xi),
\end{aligned}
$$

and further on

$$
\begin{gathered}
T_{x} \approx\left\{\begin{array}{lll}
c_{L}^{2} \frac{d \Phi_{0}^{2}}{(4 \pi \lambda)^{2}} & \text { for } & B \gg B_{c r} \\
c_{L}^{2} \frac{d \Phi_{0}^{2}}{(4 \pi \lambda)^{2}}\left(\frac{B_{c r}}{B}\right)^{1 / 2} & \text { for } & B \ll B_{c r}
\end{array}\right. \\
T_{z} \approx\left\{\begin{array}{lll}
c_{L}^{2} \frac{d \Phi_{0}^{2}}{(4 \pi \lambda)^{2}} \frac{2}{\ln \left(B / B_{c r}\right)} & \text { for } & B \gg B_{c r} \\
c_{L}^{2} \frac{d \Phi_{0}^{2}}{(4 \pi \lambda)^{2}}\left(\frac{B_{c r}}{B}\right) & \text { for } & B \ll B_{c r} .
\end{array}\right.
\end{gathered}
$$

The relative strength of fluctuations of in-plane- and $z$ bonds is different for small and large fields. At large fields $w_{x}^{\text {th }} / a_{0}^{2}$ is field-independent, whereas $w_{z}^{\text {th }} / a_{0}^{2} \sim 1 / \ln B$ increases without bounds, i.e. $w_{x}^{\text {th }} \ll w_{z}^{\text {th }}$. Thus for increasing fields $T_{x}$ saturates at a constant value, whereas $T_{z}$ vanishes. At small fields the situation is reversed: $w_{x}^{\text {th }} \gg w_{z}^{\text {th }}$ because $w_{z}^{\text {th }} / a_{0}^{2} \sim B$ and $w_{x}^{\text {th }} / a_{0}^{2} \sim B^{1 / 2}$.

The bond widths provide more information than the typical displacement $\left\langle\mathbf{u}^{2}\right\rangle^{1 / 2}$ evaluated by the usual Lindemann criterion. However, they are related by $\left\langle\mathbf{u}^{2}\right\rangle \approx$ $\max \left(w_{x}^{\text {th }}, w_{z}^{\text {th }}\right)$. In our Lindemann criterion (26) for the different types of phase transitions we use the same Lindemann number $c_{L}$. In principle these values might be different, since $z$-bonds and in-plane bonds are not not equivalent from the point of view of lattice symmetries. But a use of different Lindemann numbers would lead only to a quantitative shift of the phase boundaries, which shall not be our main concern here.

In order to associate the bond widths to possible melting and decoupling transitions, it is worth recalling the main features of these transitions. Since for anisotropies $\gamma \gg 1$ the interaction between vortices in the same layer is much weaker than the interaction of vortices in different layers one might in principle expect the possibility that melting occurs as a two-step process. In a first step the positional correlations of vortices in different layers could get lost while the correlations within the layers are preserved (at least as quasi-long-range order). This remaining two-dimensional order within the layers could then become short-ranged at a second transition at a higher temperature. However, this scenario is probably 
not realized, as one can conclude from an analogy to layered $X Y$-models. On symmetry grounds their phase diagram should be topologically equivalent to that of layered crystals. It was shown 45 that these models have only a single transition where order is destroyed in all directions. Although the relative fluctuations between the layers can become arbitrarily large for weak coupling between the layers, which is the case for layered superconductors at large fields, the transition temperature does not drop below that of the two-dimensional system. In the Lindemann approach melting has therefore to be identified with the breaking of in-plane bonds.

The breaking of $z$-bonds therefore describes a different transition, which is not related to the loss of crystalline order. Two possible transitions have been considered recently: the decoupling transition and the supersolid transition The decoupling transition of layered superconductors 27 46 describes the loss of phase coherence in the superconducting order parameter between different layers and in general does not coincide with the melting transition. The location of this transition can be estimated by a Lindemann-type criterion for the phase difference between neighboring layers. Although the vortex displacements are the main source of such phase fluctuations, a Lindemann criterion for phase differences is in general not equivalent to a Lindemann criterion for vortex displacements, since a local vortex displacement leads to a nonlocal phase distortion. However, at fields below the crossover field $B_{c r}$, both types of Lindemann criteria actually are equivalent and the decouplipgtransition is captured just by breaking of $z$-bonds.27 46 At large fields the breaking of $z$-bonds describes the location of the transition from a solid to a supersolid vortex crystal due to a proliferation of vacancy and interstitial lines in the vortex line crystal. 48

\section{B. Pinning bond width}

After the brief review on the effect of thermal fluctuations on bond widths and the possible interpretations of bond-breaking we now focus on our main issue, the contribution of pinning to the bond widths and the question how the above equilibrium phase diagram of the pure evolves into a nonequilibrium phase diagram of the disordered system.

Only very recently the equilibrium phase diagram of the disordered system $(v=0)$ has received some attention (see Refs. 32 38. In particular the breaking of inplane bonds has been used as indication for the transition from a topologically ordered phase (Bragg-glass) to a topologically disordered phase (vortex glass). For small magnetic fields the quantitative evaluation of the bond widths turned out to be quite a subtle issue because of the strong dispersion of the elastic constants in layered materials and the breakdown of perturbation theory in the low-field regime $\left(\xi \ll c_{L} a_{0}\right)$. Since we are eventu- ally interested in the dynamic effects of disorder on the dynamics, we do not attempt to reproduce the static results. However, it is worthwhile to point out that in $D=3$ the disorder induced bond fluctuations are finite for all bond types. This finiteness is found even at zero velocity and even if the bond widths are calculated from Eq. (9) within naive perturbation theory that overestimates the effect of disorder. For weak enough disorder all bonds are stable and a topologically ordered phase can persist. For low temperatures the main effect of weak disorder is to reduce the long-range translational order to a quasi-long-range order.

At sufficiently large velocities the perturbative approach, Eq. (9), can always be used to calculate the shift $\Delta T_{\alpha}$ of the bond breaking temperatures $T_{\alpha}$ with respect to the values $T_{\alpha}^{0}$ in the absence of disorder. For large velocities these temperature shifts tend to zero and the use of the lowest order perturbation theory is justified at least as long as the disorder contributions to the bond widths satisfy $w^{\text {pin }} \lesssim \xi^{2}$.

The pinning bond widths show a complicated velocity dependence with several regimes, separated by characteristic velocities related to the different elastic constants. We focus here exclusively on the high-velocity regime $v \gg c_{11} / a_{0} \eta$ for which we estimate the pinning contribution to the bond widths from Eq. (9):

$$
\begin{aligned}
& w_{x}^{\text {pin }} \approx \frac{a^{2} \Delta_{0}}{\sqrt{8 \pi} a_{0} d \xi^{3} \eta^{2} v^{2}}, \\
& w_{y}^{\text {pin }} \approx \frac{a^{2} \Delta_{0}}{4 \sqrt{2 \pi} \xi^{3} d \eta v c_{11}}, \\
& w_{z}^{\text {pin }} \approx \frac{\pi a_{0} \Delta_{0}}{16 \sqrt{2 \pi} \xi^{3} \eta v \sqrt{c_{11} c_{44}}},
\end{aligned}
$$

where we used again $c_{11} q_{\perp}^{* 2} \gg c_{44} q_{z}^{* 2}$.

From Eq. (27) one can immediately draw the important conclusion that $y$ - and $z$-bonds, for which $w^{\text {pin }}$ decays like $v^{-1}$, are affected by disorder at large velocities much stronger than $x$-bonds with $w_{x}^{\text {pin }} \sim v^{-2}$. As in $D=2$, the relative strength of $y$-bond fluctuations in comparison to $x$-bond fluctuations is in agreement with theprediction of a smectic-like flow of the vortex lattice. 0

In order to extract the net field dependence of the bond widths it is important to remember the implicit dependences of the parameters. In particular $\Delta_{0} \sim a_{0}^{-4}$ since $\Delta$ is the correlator of the pinning energy density in Eq. (4). For the same reason $\eta \sim a_{0}^{-2}$ and also all elastic constants carry a dominant implicit field dependence $\sim a_{0}^{-2}$. Thus the relative fluctuations $w_{y}^{\text {pin }} / a_{0}^{2} \sim a^{0}$ are essentially field independent, whereas $w_{x}^{\text {pin }} / a_{0}^{2} \sim w_{x}^{\text {pin }} / a_{0}^{2} \sim a_{0}^{-1}$ increase for large fields.

To visualize the velocity dependence of the dynamic transitions it is instructive to translate the pinning bond widths (27) of the driven vortex lattice into shifts $\Delta T_{x}$ of the bond-breaking temperatures (26) of the pure system. 
From $w_{\alpha}^{\text {th }}+w_{\alpha}^{\text {pin }}=c_{L}^{2} a_{0}^{2}$ the bond breaking temperature is reduced by $\Delta T_{\alpha}=-T_{\alpha} w_{\alpha}^{\mathrm{pin}} / c_{L}^{2} a_{0}^{2}$. These shifts are

$$
\begin{aligned}
& \Delta T_{x} \approx-T_{x} \frac{\epsilon_{0}^{2} \hat{\Delta}_{0}}{4 \pi c_{L}^{2} \xi^{2} \eta_{l}^{2} v^{2}}\left(\frac{B}{H_{c 2}}\right)^{1 / 2}, \\
& \Delta T_{y} \approx-T_{x} \frac{\epsilon_{0} \hat{\Delta}_{0}}{4 \sqrt{2 \pi} c_{L}^{2} \xi \eta_{l} v}, \\
& \Delta T_{z} \approx-T_{z} \frac{\pi \epsilon_{0} \hat{\Delta}_{0}}{16 \sqrt{2} c_{L}^{2} \xi \eta_{l} v}\left(\frac{B}{B_{c r}}\right)^{1 / 2} .
\end{aligned}
$$

To make the net field dependence of bond widths explicit, we have introduced a dimensionless disorder strength $\hat{\Delta}_{0}$ by $\Delta_{0}=d\left(\xi \epsilon_{0} / a_{0}^{2}\right)^{2} \hat{\Delta}_{0}$ and the friction coefficient per line $\eta_{l}=a_{0}^{2} \eta$. Furthermore we have used again the values of the elastic constants for $q_{\perp}=q_{\perp}^{*}$ and $q_{z}=q_{z}^{*}$, where $c_{11} \approx 4 c_{66} \approx \epsilon_{0} / a_{0}^{2}$, and $H_{c 2}=\Phi_{0} / 2 \pi \xi^{2}$. In terms of these parameters the high-velocity regime is restricted by $\eta_{l} v \gg \epsilon_{0} / a_{0}$.

In this high velocity regime $y$-bonds are less stable than $x$-bonds over the whole field range. Dynamic melting is thus always driven by the breaking of $y$-bonds. In the absence of disorder the $z$-bonds were more (less) stable than in-plane bonds for low (high) fields compared to $B_{c r}$. This relation is preserved in the driven case, since the bond breaking temperature of in-plane bonds is shifted more (less) than that of the $z$-bonds at low (high) fields. Thus, the topology of the phase diagram of the driven vortex lattice remains unchanged at large velocity, as one could expect naively. The high-velocity expansion does not provide evidence for a dynamic shift of the crossover field.

\section{DISCUSSION AND CONCLUSIONS}

To discuss the actual observability of the high-velocity regime it is important to compare the above velocity range to the depairing velocity of Cooper pairs. From the depairing current (see e.g. Ref. 41) the depairing velocity can be estimated as $\eta_{l} v_{\text {depair }} \sim \epsilon_{0} / \xi$. Thus even if $\xi$ is not much smaller than $a_{0}$ (as required by the validity of the perturbative approach) the high-velocity regime covers a substantial current range below the depairing current and should be experimentally accessible. As long as disorder is weak $\left(\hat{\Delta}_{0} \ll 1\right)$ the location of the dynamic melting and decoupling transitions is close to the location of the equilibrium transition of the pure system, i.e. $\Delta T_{\alpha} \ll T_{\alpha}$.

In this high-velocity regime dynamic melting is always driven by the breaking of $y$-bonds. The dynamic shifts of both melting and decoupling transition temperatures scale like $\Delta T_{\alpha} \sim v^{-1}$. This dependence is in agreement with experiment 18 and numerical simulations 19.14 and the original concept of the shaking temperature. $\mathrm{We}$ have extended this original approach, which was focused on the dynamic response of a single vortex and on the response of the lattice at large scales, on the fluctuations of bonds. Thereby we were able to put the characterization of the nonequilibrium transitions on a common basis with the Lindemann approaches to the equilibrium counterparts.

At smaller drive the velocity dependence of the bond widths and the temperature shifts will be in general weaker than at high drive. In two dimensions the velocity dependence becomes logarithmic at small velocities and the crossover between different regimes has been calculated numerically (see Fig. 11). In three dimensions there are even more dynamic regimes between the asymptotic regimes of zero and large velocity because of the complex elastic properties of the vortex lattice.

However, from Eq. 28) we can conclude that for weak disorder $\left(\hat{\Delta}_{0} \ll 1\right)$ the scaling of the high-velocity regime should be observable in a considerable velocity range below depairing. In this case, where the temperature shifts are small $\left(\Delta T_{\alpha} \ll T_{\alpha}\right)$, it should be possible to observe a dynamic shift of the phase boundaries experimentally. To the best of our knowledge, such dynamic shifts have not been reported. We naturally expect a dynamic shift to be most pronounced in the vicinity of the critical point of the melting line since the main effect of a driving current is to reduce the effective strength of disorder and this point has been found to be quite sensitive to the strength of disorder in equilibrium 23 In particular the location of the critical point will be shifted to larger fields by a driving current.

The anisotropy of bond fluctuations found above is consistent with the picture of smectic vortex flow H. $^{-1}$ due to the dominant proliferation of dislocations with Burger's vectors parallel to the drift velocity, providing the actual mechanism for bond breaking. We expect the anisotropy of bond fluctuations to have immediate consequences for the structure of the coherently flowing vortex lattice. In particular even the Bravais basis of the vortex lattice will display this anisotropy. In the presence of disorder the vortex system will show an expansion of its lattice constants similar to a thermal expansion. From the anisotropy of the bond fluctuations one has to expect that this expansion will be larger in $y$-direction than in $x$-direction. It would be interesting to analyze neutron scattering and Bitter decoration experiments on the driven vortex lattice in view of a dynamic shift of the location of the (quasi)-Bragg peaks.

\section{ACKNOWLEDGMENTS}

The authors gratefully acknowledge useful discussions with J. Kierfeld, H. Nordborg, and in particular with A.E. Koshelev.

This work was supported from Argonne National Laboratory through the U.S. Department of Energy, BESMaterial Sciences, under contract No. W-31-109-ENG-38 
and by the NSF-Office of Science and Technology Centers under contract No. DMR91-20000 Science and Technology Center for Superconductivity. S. acknowledges support by the Deutsche Forschungsgemeinschaft project SFB341 and grant SCHE/513/2-1.

${ }^{1}$ A.E. Koshelev and V. M. Vinokur, Phys. Rev. Lett. 73, 3580 (1994).

${ }^{2}$ L. Balents and M. P. A. Fisher, Phys. Rev. Lett. 75, 4270 (1995)

3 T. Giamarchi and P. Le Doussal, Phys. Rev. Lett. 76, 3408 (1996); ibid. 78, 752 (1997).

${ }^{4}$ L.W. Chen, L. Balents, M.P.A. Fisher, and M.C. Marchetti, Phys. Rev. B 54, 12798 (1996).

${ }^{5}$ L. Balents, C. Marchetti and L. Radzihovsky, Phys. Rev. Lett. 78, 751 (1997).

${ }^{6}$ S. Scheidl and V.M. Vinokur, Phys. Rev. B 56, 8522 (1997).

${ }^{7}$ L. Balents, M.C. Marchetti, and L. Radzihovsky, preprint cond-mat/9707302.

${ }^{8}$ T. Giamarchi, and P. Le Doussal, preprint condmat/9708085.

${ }^{9}$ S. Scheidl and V.M. Vinokur, preprint cond-mat/9708222.

${ }^{10}$ K. Moon, R.T. Scalettar and G.T. Zimányi, Phys. Rev. Lett. 77, 2778 (1996).

${ }^{11}$ M.C. Faleski, M.C. Marchetti, and A.A. Middleton, Phys. Rev. B 54, 12427 (1996).

12 S. Ryu, M. Hellerqvist, S. Doniach, A. Kapitulnik and D. Stroud, Phys. Rev. Lett. 77, 5114 (1996).

${ }^{13}$ S. Spencer and H.J. Jensen, Phys. Rev. B 55, 8473 (1997).

${ }^{14}$ A. Van Otterlo, R.T. Scalettar and G.T. Zimányi, unpublished.

15 S. Bhattacharya and M.J. Higgins, Phys. Rev. Lett. 70, 2617 (1993).

${ }^{16}$ W.K. Kwok et al., Phys. Rev. Lett. 73, 2614 (1994).

${ }^{17}$ U. Yaron et al., Nature 376, 753 (1995).

18 M. C. Hellerqvist, et al., Phys. Rev. Lett. 76, 2617 (1996).

19 M.J. Higgins and S. Bhattacharya, Physica C 257, 232 (1996), and references therein.

${ }^{20}$ M. Marchevsky et al., Phys. Rev. Lett. 78, 531 (1997).

${ }^{21}$ F. Pardo et al., preprint.

${ }^{22}$ E. Zeldov et al., Nature 375, 373 (1995).

${ }^{23}$ B. Khaykovich et al., Phys. Rev. B —bf 56, R517 (1997).

${ }^{24}$ D.R. Nelson and H.S. Seung, Phys. Rev. B 39, 9153 (1989).

25 A. Houghton, R.A. Pelcovits, and A. Sudbo, Phys. Rev. B 40, 6763 (1989).

${ }^{26}$ E.H. Brandt, Phys. Rev. Lett. 63, 1106 (1989).

${ }^{27}$ L.I. Glazman and A.E. Koshelev, Phys. Rev. B 43, 2835 (1991).

${ }^{28}$ G. Blatter, V. Geshkenbein, A. Larkin, and H. Nordborg, Phys. Rev. B 54, 72 (1996).

29 T. Nattermann, Phys. Rev. Lett. 64, 2454 (1990).

30 T. Giamarchi and P. Le Doussal, Phys. Rev. Lett. 72, 1530 (1994); Phys. Rev. B 52, 1242 (1995).

31 A.I. Larkin and V.M. Vinokur, Phys. Rev. Lett. 75, 4666 (1995).
32 J. Kierfeld, T. Nattermann, and T. Hwa, Phys. Rev. B 55, 626 (1997).

33 D. Ertas and D.R. Nelson, Physica C 27279 (1996).

${ }^{34}$ V. M. Vinokur et al., Preprint, (1997).

35 J. Kierfeld, preprint cond-mat/9609045.

36 D.S. Fisher, Phys. Rev. Lett. 78, 1964 (1997).

37 T. Giamarchi, and P.Le Doussal, Phys. Rev. B 55, 6577 (1997).

38 A.E. Koshelev and V.M. Vinokur, preprint (1997).

${ }^{39}$ A. Schmid and W. Hauger, J. Low Temp. Phys. 11, 667 (1973).

40 J.M. Kosterlitz and D.J. Thouless, J. Phys. C 6, 1181 (1973).

${ }^{41}$ G. Blatter, M.V. Feigel'man, V.B. Geshkenbein, A.I. Larkin and V.M. Vinokur, Rev. Mod. Phys. 66, 1125 (1994).

42 J.L. Cardy and S. Ostlund, Phys. Rev. B 25, 6899 (1982).

43 J. Villain and J.F. Fernandez, Z. Phys. B 54, 139 (1984).

${ }^{44}$ D.S. Fisher, M.P.A. Fisher, and D.A. Huse, Phys. Rev. B 43, 130 (1991).

${ }^{45}$ S. Hikami and T. Tsuneto, Prog. Theor. Phys. 63, 387 (1989); J. Friedel, J. Phys. Condensed Matter, 1, 7757 (1989); S.E. Korshunov, Eurephys. Lett. 11, 757 (1990); S.W. Pierson and O.T. Valls Phys. Rev. B 45, 13076 (1992).

${ }^{46}$ L.L. Daemen, L.N. Bulaevskii, M.P. Maley, and J,Y, Coulter, Phys. Rev. B 47, 11291 (1993).

47 A.E. Koshelev, L.I. Glazman, and A.I. Larkin, Phys. Rev. B 53, 2786 (1996).

${ }^{48}$ E. Frey, D.R. Nelson, and D.S. Fisher, Phys. Rev. B 49, 9723 (1994); see also M. Feigel'man, V.B. Geshkenbein, and A.I. Larkin, Physica C 167, 177 (1990).

${ }^{49}$ I. Aranson, A.E. Koshelev, and V.M. Vinokur, Phys. Rev. B 56, 5136 (1997). 


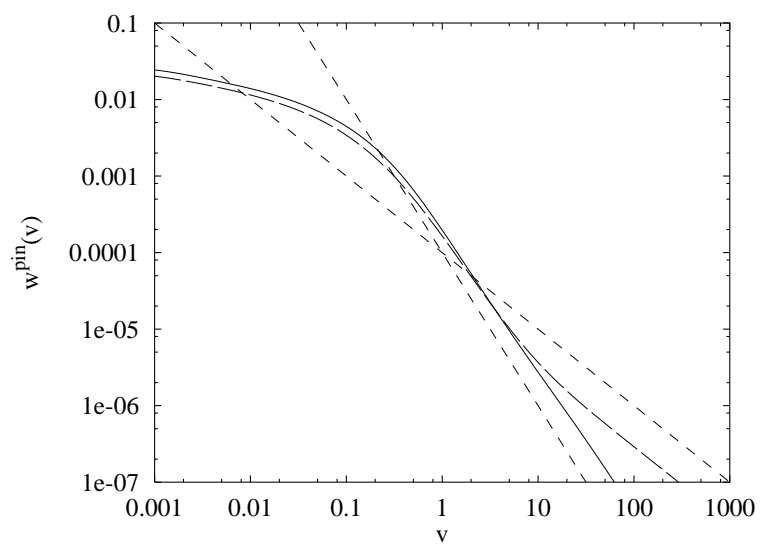

FIG. 1. Plot of pinning bond widths $w_{x}^{\text {pin }}$ (full line) and $w_{y}^{\text {pin }}$ (long dashed line) calculated numerically from Eq. (9) for the parameters specified in the text. The short dashed lines represent dependences $w^{\text {pin }}(v) \sim v^{-1}, v^{-2}$, which are realized at high $v$.

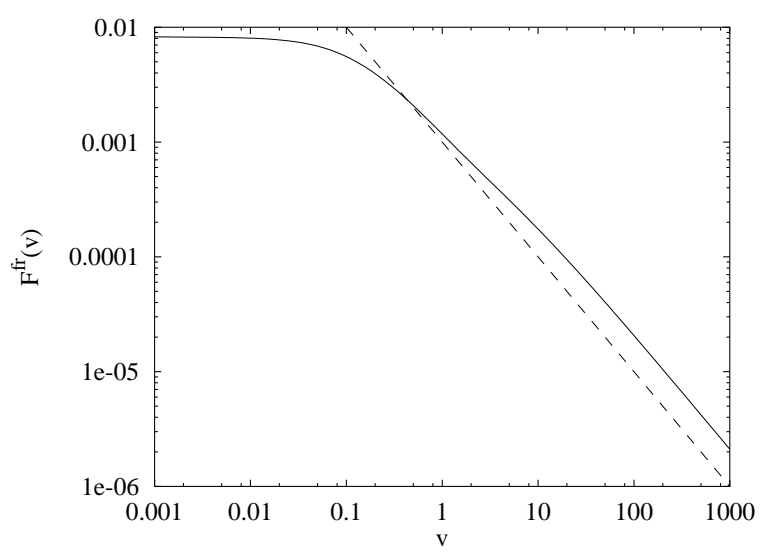

FIG. 2. Plot of friction force (full line) calculated numerically from Eq. (11) for the parameters specified in the text. The dashed line represent the dependence $F^{\mathrm{fr}}(v) \sim v^{-1}$ at high $v$.

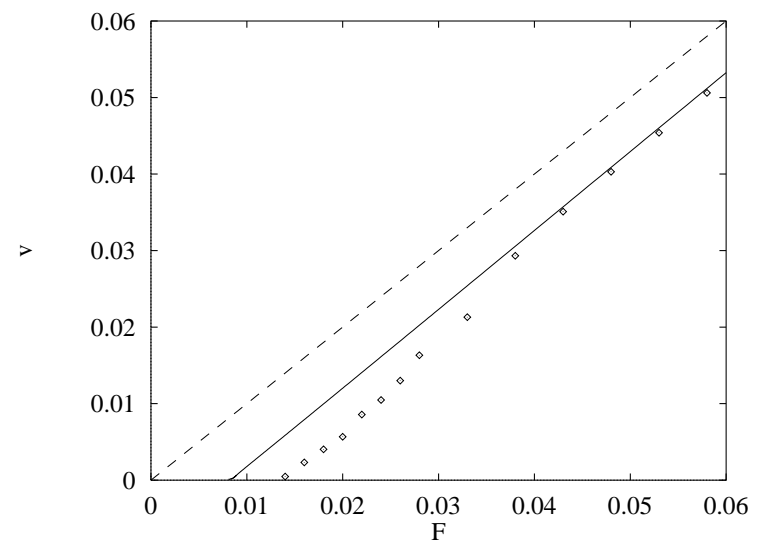

FIG. 3. Plot of the transport characteristic resulting from Eq. (11), full line, and the simulation data of Ref. 11 (dashed line).

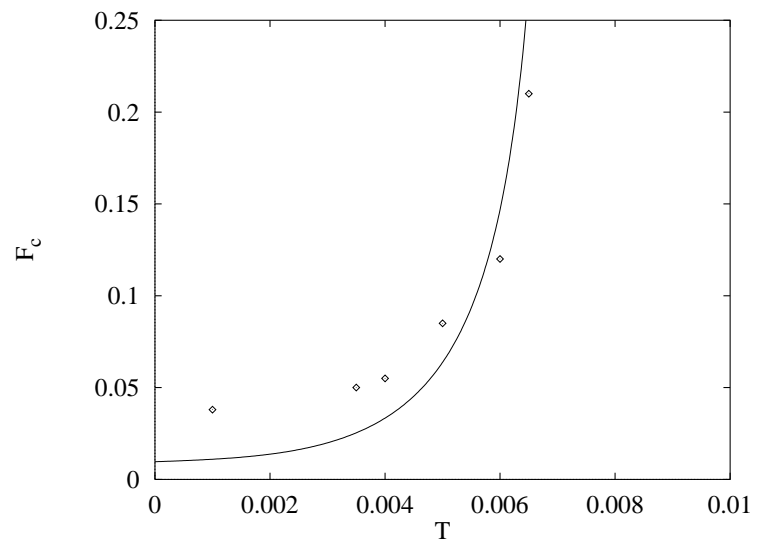

FIG. 4. Plot of critical force for the transition from coherent to incoherent vortex motion. The full line is our result from the Lindemann criterion, the dots are simulation results from Ref. 1 . 